\title{
3-D numerical simulations of rotating jets
}

\section{The case of the DG Tau microjet}

\author{
A. H. Cerqueira ${ }^{1}$ and E. M. de Gouveia Dal Pino ${ }^{2}$ \\ ${ }^{1}$ LATO-DCET-UESC, Rodovia Ilhéus - Itabuna, km 16, Ilhéus, Bahia, CEP 45662-260, Brazil \\ e-mail: hoth@uesc.br \\ 2 IAG-USP, Rua do Matão, 1226, Cidade Universitária, São Paulo, São Paulo, CEP 01060-970, Brazil \\ e-mail: dalpino@astro.iag.usp.br
}

Received 29 April 2004 / Accepted 6 September 2004

\begin{abstract}
We here present results of three-dimensional Smoothed Particle hydro and magnetohydrodynamics simulations of rotating jets, also including the effects of radiative cooling, precession and velocity variability. Using initial conditions and parameters which are particularly suitable for the DG Tau microjet, we have been able to approximately reproduce its complex knotty morphology and kinematics. We have also obtained radial velocity maps which are in good agreement with the data obtained by Bacciotti et al., thus indicating that their interpretation that the DG Tau microjet is rotating is correct. Finally, we have found that a magnetic field of the order of $\approx 0.5 \mathrm{mG}$ is sufficient to collimate the jet against the lateral expansion that is caused by the centrifugal forces.
\end{abstract}

Key words. ISM: jets and outflows: Herbig-Haro objects - stars: formation

\section{Introduction}

The seek for the real nature of the accretion-ejection mechanism underlying the formation of the jets is one of the major contributions of the detailed analysis of Herbig-Haro $(\mathrm{HH})$ jet data. The statement that MHD models can be responsible for generating the jets associated with Young Stellar Objects is still a matter of debate. The theoretical discussion of the so-called magneto-centrifugal models for jet launching (e.g., Blandford \& Payne 1982; Königl 1982; Spruit 1996), can be strongly constrained by the existence of observational data.

Recent observations of microjets associated with T-Tauri stars, namely, DG Tau, RW Aur, TH28 and LkH $\alpha 321$ (Bacciotti et al. 2002; Coffey et al. 2004), have revealed important trends in the jet radial velocity field that are consistent with a rotation pattern inside the jet beam. The presence of rotation could be understood in the context of the magneto-centrifugal models as due to jet launching from a Keplerian accretion disk along with the magnetic field lines which are anchored into the disk-star system and provide the collimation of the beam.

Motivated by these recent findings, we have carried out a set of fully three-dimensional simulations of rotating jets, also taking into account the presence of radiative cooling, jet precession, and velocity variability (to allow the production of internal knots). All these are necessary ingredients to address the kinematical complexity of the microjets and we are here particularly interested in the DG Tau microjet case, which is one of the best studied in the literature. We have also investigated, in few models, the collimating effects of magnetic fields upon rotating jets.

\section{Numerical method and the simulated models}

In order to examine the behaviour of the microjets and their early evolution near the source under the effects of both rotation and precession, we here employ modified versions of our threedimensional Smoothed Particle Hydrodynamics (3-D SPH) and Magnetohydrodynamics (3-D SPMHD) codes which were previously designed to investigate the large scale structure and evolution of protostellar jets either in the absence (see, e.g., de Gouveia Dal Pino 2001) or in the presence of magnetic fields (e.g, Cerqueira \& de Gouveia Dal Pino 2001).

Our computational domain is a rectangular box that mimics the ambient medium and has dimensions $-25 R_{\mathrm{j}} \leq x \leq 25 R_{\mathrm{j}}$, and $-10 R_{\mathrm{j}} \leq y, z \leq 10 R_{\mathrm{j}}$, where $R_{\mathrm{j}}$ is the initial jet radius (which is also the code distance unit). The Cartesian coordinate system has its origin at the center of the box and the jet flow is continuously injected into the bottom of the box [at the inlet which has coordinates $\left.\boldsymbol{r}=\left(-25 R_{\mathrm{j}}, 0,0\right)\right]$ with top-hat density and pressure profiles. The jet and gas pressures are assumed to be in equilibrium at the inlet. Inside the box, the SPH particles are initially regularly distributed in a cubic lattice. An outflow boundary condition is assumed for the boundaries of the box. The particles are smoothed out by a spherically symmetric kernel function of width $h$, and the initial values of $h$ were chosen 
to be $0.4 R_{\mathrm{j}}$ and $0.2 R_{\mathrm{j}}$ for the ambient and jet particles, respectively. The adiabatic index of the ambient medium and the jet is assumed to be $\gamma=5 / 3$, and an ideal equation of state is used. The radiative cooling, due to collisional excitation and recombination, is implicitly calculated using a time-independent cooling function for a gas of cosmic abundances cooling from $T \simeq 10^{6}$ to $9 \times 10^{3} \mathrm{~K}$.

The adopted parameters for the simulations are appropriate to the conditions generally found in protostellar jets and, in particular, in the DG Tau microjet. We take a number density ratio between the jet and the ambient medium $\eta=n_{\mathrm{j}} / n_{\mathrm{a}}=10 ; n_{\mathrm{j}}=$ $2000 \mathrm{~cm}^{-3}$; an average ambient Mach number $M_{\mathrm{a}}=v_{\mathrm{j}} / c_{\mathrm{a}}=19$, where $v_{\mathrm{j}} \simeq 300 \mathrm{~km} \mathrm{~s}^{-1}$ is the average jet velocity of DG Tau (e.g., Bacciotti et al. 2002; Pyo et al. 2003), and $c_{\mathrm{a}} \simeq 16 \mathrm{~km} \mathrm{~s}^{-1}$ is the ambient sound speed for a gas with an average temperature of $10^{4} \mathrm{~K}$; and $R_{\mathrm{j}}=5.6 \times 10^{14} \mathrm{~cm}$ (see e.g., Dougados et al. 2000; Bacciotti et al. 2002).

We have carried out several simulations considering both continuous and pulsing jets (see below). In the latter cases, we have adopted a sinusoidal profile to describe the ejection velocity time-variability at the inlet: $v_{\mathrm{o}}(t)=v_{\mathrm{j}}\left[1+A \cdot \sin \left(\frac{2 \pi}{\tau_{\text {pul }}} t\right)\right]$, where $A$ is the velocity amplitude and $\tau_{\text {pul }}$ is the period of the oscillation. The values for these parameters were obtained from observations of the DG Tau microjet (see, e.g., Lavalley et al. 1997; Raga et al. 2001) which suggest $A=0.33$, and $\tau_{\text {pul }}=8$ years $=0.71 t_{\mathrm{d}}$ (where $t_{\mathrm{d}}=R_{\mathrm{j}} / c_{\mathrm{a}} \approx 11.3$ years corresponds to the transverse jet dynamical time). In the numerical models where the jet precession has been also taken into account, we have assumed an equal precession period (i.e., $\tau_{\text {prec }}=\tau_{\text {pul }}=8$ years) and a precession half-angle $\theta=5^{\circ}$ as indicated by the DG Tau jet observations (Lavalley-Fouquet et al. 2000; Dougados et al. 2000).

For the computation of the jet rotation around its main axis, we have assumed that the flow conserves its angular momentum after its passage through the Alfven surface (i.e., $v_{\phi} r=$ constant, where $v_{\phi}$ is the toroidal velocity in the normal direction to the jet axis). This is consistent with magneto-centrifugal mechanisms for jet launching (see, e.g., Spruit 1996) and is also suggested by the observations of the DG Tau microjet (Bacciotti et al. 2002) which imply $v_{\phi}=\left(4.362 \times 10^{20} / r\right) \mathrm{cm} \mathrm{s}^{-1}$ where $r$ is the radial distance to the jet axis in $\mathrm{cm}$. This gives $v_{\phi} \simeq 8 \mathrm{~km} \mathrm{~s}^{-1}$ at the jet surface $\left(r=R_{\mathrm{j}}=5.6 \times 10^{14} \mathrm{~cm}\right)$, and $v_{\phi} \approx 55 \mathrm{~km} \mathrm{~s}^{-1}$ near the jet axis (at $\left.r \simeq 0.15 R_{\mathrm{j}}\right)^{1}$. It should be emphasized that we are here mostly interested in carrying out simulations of jet regions near the source but beyond the Alfven surface (e.g., Spruit 1996) where the flow has accelerated to velocities larger than the Alfven speed and is believed to be already collimated, and which are, at least in some cases, partially resolved by optical observations (as for instance, DG Tau).

Table 1 summarizes the properties of the simulated models. In model $\mathrm{A}$, we have run a precessing jet with constant velocity at injection and no rotation. In model $\mathrm{B}$, we have

${ }^{1}$ We notice that the code does not allow, by construction, any SPH particle to occupy the jet axis, so that the equation above for $v_{\phi}$ has no singularities, as it is employed only for values of $r$ which are larger than zero.
Table 1. The jet models.

\begin{tabular}{ccccc}
\hline \hline Model & $\tau_{\text {pul }}$ & $\tau_{\text {prec }}$ & Rotation? & $B$-field? \\
\hline A & - & 8 years & no & no \\
B & 8 years & 8 years & no & no \\
C & 8 years & 8 years & yes & no \\
D & - & - & yes & no \\
E & - & - & yes & yes \\
\hline
\end{tabular}

considered a pulsing precessing jet also without rotation, and in model $\mathrm{C}$ we have run a jet which is simultaneously pulsating, precessing, and rotating. All these three models are purely hydrodynamical and share the same initial conditions as described above. Their velocity distribution is compared in Fig. 1 after they have propagated over a time $t / t_{\mathrm{d}}=2.5$. In model $\mathrm{C}$, the jet is precessing counter clockwise while rotating clockwise. The jet of model A develops a V-shape structure at the head which is commonly detected in precessing jets (e.g., Masciadri et al. 2002; Masciadri \& Raga 2003; Rosen \& Smith 2004) and is due to the impact of the parcels propagating in different directions in the wiggling flow with the bow-shock front. In the jet of model B, the combined effects of pulsation and precession produce internal working surfaces propagating in different directions. In this case, a narrower bowshock structure develops at the head that makes it to propagate slightly faster than the jet of model A. The rotating jet of model $\mathrm{C}$ essentially bears the same features of the previous models but develops an even larger head than model A due to the centrifugal forces that tend to push the jet flow outwards in the radial direction (see also Fig. 3 and discussion below).

Let us now concentrate on the early evolution of the rotating, pulsing, precessing jet of Fig. 1c (model C) whose characteristics qualitatively resemble those of the DG Tau microjet (Dougados et al. 2000; Bacciotti et al. 2002). Figure 2a shows the jet of Fig. 1c at an earlier time that corresponds to the inferred dynamical age of the DG Tau microjet $(\sim 11.3 \mathrm{yr}$; see e.g., Dougados et al. 2000; Lavalley et al. 1997; Pyo et al. 2003). The density contour plot presents nearly similar features to those observed in the DG Tau microjet: an elongated, highly collimated beam near the jet base, a slight sideway displacement of the beam that has been previously interpreted as an evidence for precession of the jet axis, and a more prominent bow shock (at $\approx-8 R_{\mathrm{j}}$ in Fig. 2 a) which can be compared with the B1 knot of DG Tau (Dougados et al. 2000; Lavalley-Fouquet et al. 2000).

For a comparison with the radial velocities obtained from the HST observations of DG Tau by Bacciotti et al. (2002), in Fig. $2 b$ we have built diagrams of the radial velocity across the jet (taken at an angle of $45^{\circ}$ with respect to the jet axis, in order to mimic the DG Tau inclination with respect to the line of sight) in four different slices along the flow which are separated by $0.5 R_{\mathrm{j}} \simeq 20 \mathrm{AU}$ and are marked in Fig. 2a. [These correspond to the same slices examined in Fig. 1 of Bacciotti et al.: $x=-24.5 R_{\mathrm{j}}$ (Position I), $-24 R_{\mathrm{j}}$ (Position II), $-23.5 R_{\mathrm{j}}$ (Position III) and $-23.0 R_{\mathrm{j}}$ (Position IV).] Figure $2 \mathrm{c}$ depicts, for each diagram of Fig. $2 b$, the radial velocity differences 

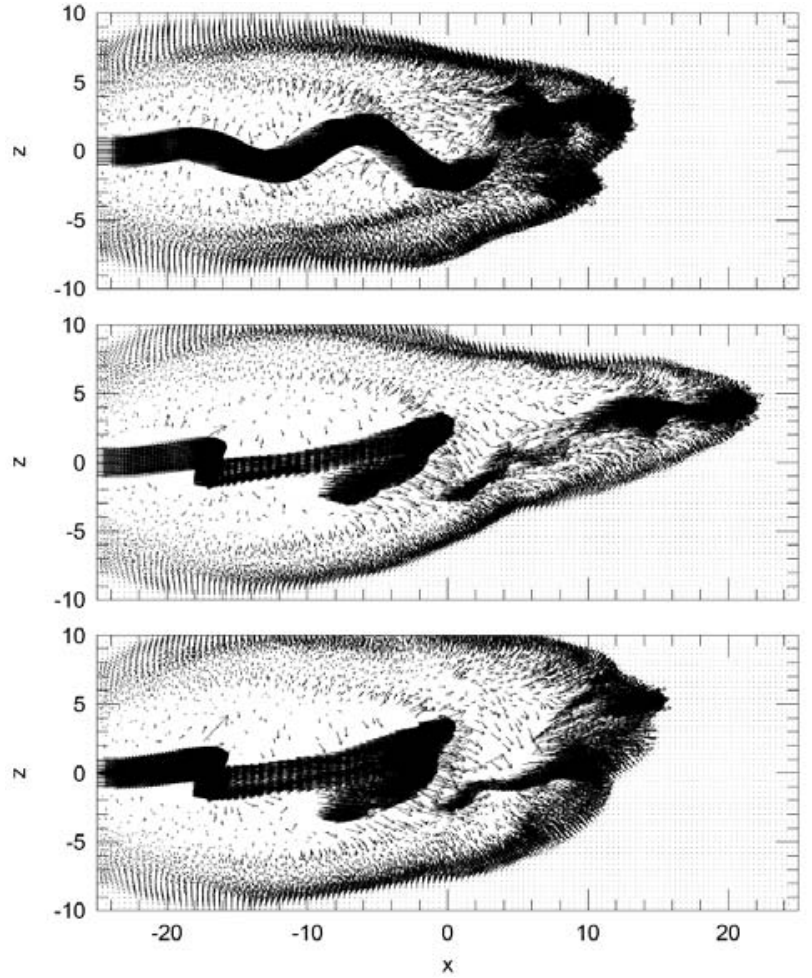

Fig. 1. Midplane velocity field distribution for the jet models A (top), $\mathrm{B}$ (middle) and C (bottom), at $t / t_{\mathrm{d}}=2.5$. See the text for details.

between positions which are symmetric with respect to the central region of the jet, as a function of the distance from the jet axis. We notice that the differences in radial velocity are negative, therefore indicating that the net velocity is oriented towards the $-y$ direction. These negative shifts in velocity are obviously a consequence of the fact that the jet of model $\mathrm{C}$ is rotating clockwise. As expected, these shifts are null in the jets that are only precessing, like those of Figs. 1a and 1b. The resulting radial velocity shifts in Fig. $2 \mathrm{c}, \Delta v_{\mathrm{r}} \lesssim-15 \mathrm{~km} \mathrm{~s}^{-1}$, are very similar to those obtained by Bacciotti et al. which are approximately reproduced in the figure by the solid lines. We note that these solid lines also reproduce the results recently obtained by Pesenti et al. (2004) assuming that the DG Tau microjet is launched magnetocentrifugally by a warm accretion disk. The consistency between the present results and their predictions is an additional support for the magnetocentrifugal mechanism for jet launching.

Finally, aiming to check the collimation effects of the magnetic fields in rotating jets, we have performed simulations of continuous, non-precessing flows under the presence of helical fields. Given the present uncertainties related to the real orientation and strength of the magnetic fields in protostellar jets we have assumed, as in previous work (e.g., Cerqueira \& de Gouveia Dal Pino 2001) a force-free helical magnetic field which also extends to the ambient medium and whose radial functional dependences for the toroidal and longitudinal components are respectively given by:

$B_{\phi}(r)=B_{0}[f(r) d]^{1 / 2}$,

$B_{x}(r)=B_{0}[1-f(r)(r+d)]^{1 / 2}$,
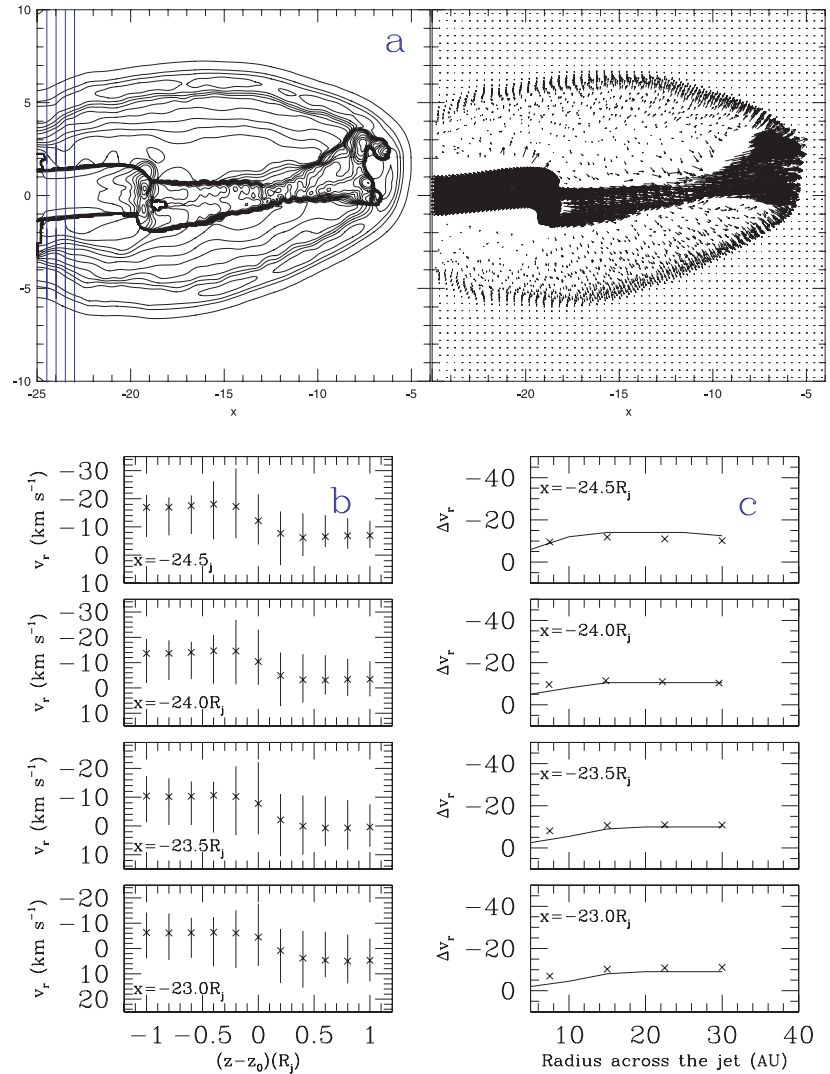

Fig. 2. a) Midplane density contour (left panel) and velocity field (rigth panel) distributions for the model C (see Table 1), at $t / t_{\mathrm{d}}=1$ which approximately corresponds to the dynamical age of the DG Tau microjet; b) average radial velocities across the jet for the different positions along the beam that are marked in Fig. 2a, above. We notice that the jet axis is actually located at $z_{0}=0.1 R_{\mathrm{j}}$ due to the effects of the precession. The vertical bars indicate the maximum and minimum values of the radial velocities obtained for each radial distance (the distances are in units of the jet radius); c) radial velocity differences between positions across the jet which are symmetric with respect to the jet center, as a function of the radial distance. Crosses: our results. Full line: an approximate fitting to Bacciotti et al. (2002) observations and Pesenti et al. (2004) results (the distances are in units of AU and the velocities in units of $\mathrm{km} \mathrm{s}^{-1}$ ).

where $r=\sqrt{y^{2}+z^{2}}$ is the radial distance from the jet axis, $f(r)=0.5 C r^{2} /(r+0.5 d)^{3}$, and the constants $C$ and $d$ are assumed to be 100 and $3 R_{\mathrm{j}}$, respectively. In these equations, $B_{0}$ gives the maximum strength of the magnetic field and corresponds to the magnitude of the longitudinal component at the jet axis and the magnitude of the toroidal component at $r=3 R_{\mathrm{j}}$ (see Cerqueira \& de Gouveia Dal Pino 2001).

As an example, Fig. 3 compares the early evolution of one of these MHD runs (model E of Table 1) with a purely hydrodynamical jet (model D of Table 1). Both have the same initial conditions of the previous models which are appropriate to DG Tau microjet. The MHD model has an initial value of the ratio between the magnetic and the gas pressure $\beta=p_{\text {mag }} / p_{\text {th }}=$ $B^{2} /(8 \pi p)=10$ near the jet surface which corresponds to a magnetic field intensity $\sim 0.5 \mathrm{mG}$. The centrifugal force upon the fluid in the radial directions causes the beam radius of the hydrodynamical jet to increase as the central parts of the flow tend 

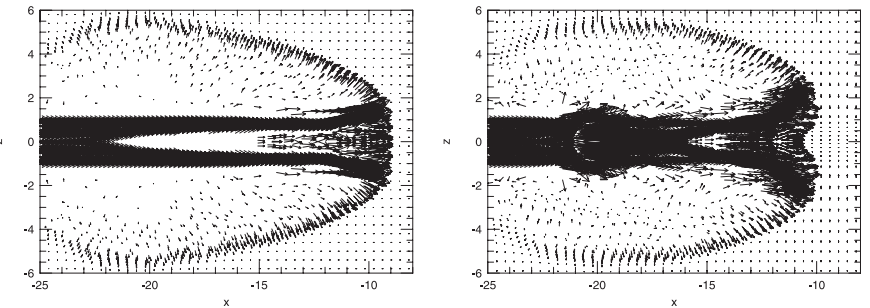

Fig. 3. Midplane velocity field distributions for the purely hydrodynamical model D (left) and for the MHD model E (right) of Table 1, at $t / t_{\mathrm{d}}=1$. The distances are in units of $R_{\mathrm{j}}(\approx 38 \mathrm{AU})$.

to move laterally outwards. Though less obvious, we have previously noticed the same unbalancing of radial forces and jet enlargement in the rotating jet of Fig. 1c (model C), particularly in its head. When a helical magnetic field (with surface $\beta \simeq 10$ ) is introduced in the system, we find that the tension force (or hoop stress) associated to the toroidal component of the magnetic field is able to collimate the jet. Smaller values of the surface $\beta$ were found to be unable to prevent the lateral expansion and similar results were also obtained for pulsing jets under the same conditions (see discussion below).

\section{Discussion and conclusions}

In the present work, we have explored the role of rotation on the evolution of YSO microjets addressing, in particular, the DG Tau microjet that has recently shown strong observational evidence for rotation (Bacciotti et al. 2002). With the help of 3 -D hydro and magnetohydrodynamical simulations, we have investigated several models that also included the effects of precession and pulsation of the flow. Our main purpose here was to verify through fully 3-D simulations whether the signature of rotation was really unique and unmistakable in the jet flow when other effects, like precession and pulsation, were also considered. The main conclusions of this study can be summarized as follows:

1. The morphology and kinematics of the DG Tau microjet was best reproduced in our simulations by model $\mathrm{C}$ (Figs. 1c and 2a), which presents a jet with a sinusoidal velocity variability (with mean velocity of $300 \mathrm{~km} \mathrm{~s}^{-1}$, halfamplitude of $100 \mathrm{~km} \mathrm{~s}^{-1}$, and a period of $8 \mathrm{yr}$ ), a precession of the outflow axis (with half-angle of $5^{\circ}$ and $8 \mathrm{yr}$ period), and a rotation around its axis with a velocity $v_{\phi} \simeq 8 \mathrm{~km} \mathrm{~s}^{-1}$ at the jet surface, and $\approx 55 \mathrm{~km} \mathrm{~s}^{-1}$ near the jet axis,. These latter conditions satisfy angular momentum conservation and are consistent with magneto-centrifugal jet launching models. As remarked before, these characteristics approximately match those suggested by the observations reported in the literature. We note also that previous numerical studies of the morphology and kinematics of this object (e.g., Raga et al. 2001) have obtained similar conclusions although they have not taken into account the effects of rotation.

2. The simulated radial velocities for model $\mathrm{C}$ have revealed good agreement with the data obtained by Bacciotti et al. (2002), therefore suggesting that their interpretation that the DG Tau microjet is rotating must be correct.
3. The inclusion in the models of a helical magnetic field permeating the jet and the ambient medium, with a maximum value of $\beta \simeq 10$ near the jet surface (corresponding to a toroidal magnetic field $\sim 0.5 \mathrm{mG}$ ), has provided a collimation of the outflow which otherwise would tend to expand laterally while propagating downstream (Fig. 3). Though the MHD models here investigated were only simple cases involving rotating continuous (or pulsing) jets without precession, the results above suggest that the presence of magnetic fields of the order of $\sim 0.5 \mathrm{mG}$ in the DG Tau microjet would be sufficient to collimate the beam against the centrifugal forces. In fact, a simple estimate of the magnetic tension forces that would be required to balance the centrifugal forces at the DG Tau microjet surface gives a magnetic field strength $B=\left(4 \pi \rho v_{\phi}^{2}\right)^{1 / 2} \sim 0.2 \mathrm{mG}$ (where $\rho \simeq n_{\mathrm{j}} m_{\mathrm{H}} \sim 3.3 \times 10^{-21} \mathrm{~g} \mathrm{~cm}^{-3}$ and $\left.v_{\phi}=8 \mathrm{~km} \mathrm{~s}^{-1}\right)$, which is compatible with the required value above from the simulations. We further notice that this would be the expected strength of the magnetic field at radial distances $\sim 40 \mathrm{AU}$ from the source. Magnetic flux conservation would then imply strengths $\sim 100 \mathrm{G}$ near the stellar surface which are compatible both with the values expected for protostellar sources and also with magneto-centrifugal mechanisms for jet production.

Acknowledgements. We are indebted to Francesca Bacciotti and an anonymous referee for their very helpful comments and suggestions. We also acknowledge partial support of the Brazilian agencies FAPESB, FAPESP and CNPq, the Milenium Institute, and the projects PROPP-UESC (00220.1300.327) and PRODOC- UFBa (991042-88).

\section{References}

Bacciotti, F., Ray, T. P., Mundt, R., Eislöffel, J., \& Solf, J. 2002, ApJ, 576, 222

Blandford, R. D., \& Payne, D. G. 1982, MNRAS, 199, 1982

Cerqueira, A. H., \& de Gouveia Dal Pino, E. M. 2001, ApJ, 560, 779

Coffey, D., Bacciotti, F., Woitas, J., Ray, T. P., \& Eislöffel, J. 2004, ApJ, 604, 758

de Gouveia Dal Pino, E. M. 2001, ApJ, 551, 347

Dougados, C., Cabrit, S., Lavalley, C., \& Ménard, F. 2000, A\&A, 357, L61

Königl, A. 1982, ApJ, 261, 115

Lavalley, C., Cabrit, S., Dougados, C., Ferruit, P., \& Bacon, R. 1997, A\&A, 327, 671

Lavalley-Fouquet, C., Cabrit, S., \& Dougados, C. 2000, A\&A, 356, L41

Masciadri, E., \& Raga, A. C. 2003, Rev. Mex. Astron. Astrofis., 15, 140

Masciadri, E., de Gouveia Dal Pino, E. M., Raga, A. C., \& Noriega-Crespo, A. 2002, ApJ, 580, 950

Pesenti, N., Dougados, C., Cabrit, S., et al. 2004, A\&A, 416, L9

Pyo, T.-S., Kobayashi, N., Hayashi, M., et al. 2003, ApJ, 590, 340

Raga, A. C., Cabrit, S., Dougados, C., \& Lavalley, C. 2001, A\&A, 367, 959

Reipurth, B., \& Bally, J. 2001, ARA\&A, 39, 403

Rosen, A., \& Smith, M. D. 2004, MNRAS, 347, 1097

Spruit, H. C. 1996, in Evolutionary processes in binary stars, NATO ASI Ser. C, 477, 249 